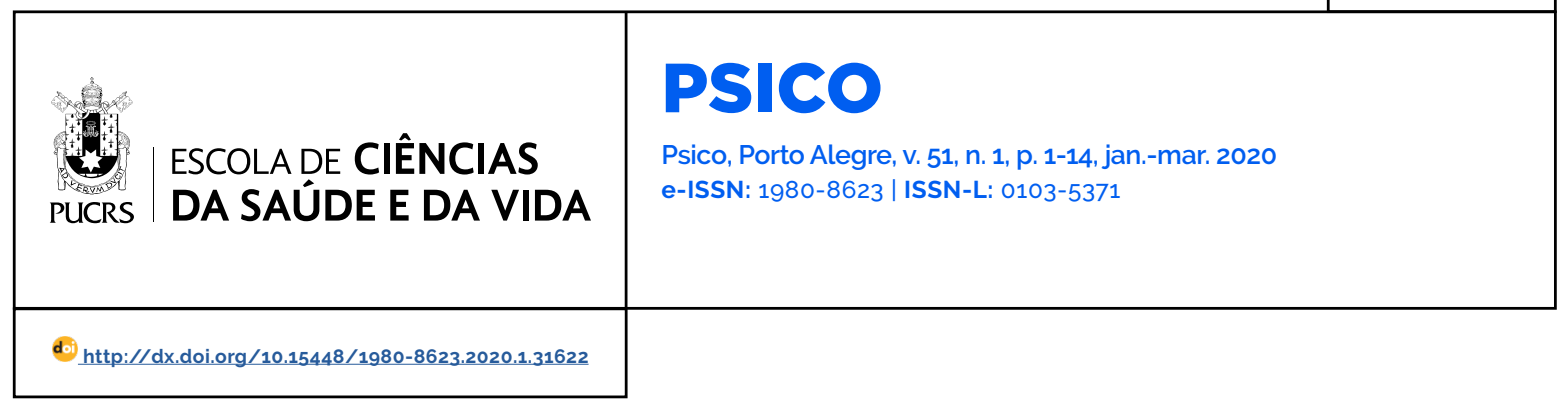

ARTIGOS

\title{
Escolaridade materna e indicadores desenvolvimentais na criança: mediação do conhecimento materno sobre o desenvolvimento infantil
}

\author{
Maternal education and developmental indicators in children: mediation of maternal \\ knowledge on child development \\ Escolaridad materna e indicadores de desarrollo em niños: mediación del \\ conocimiento de la madre sobre el desarrollo infantil
}

\author{
Patricia Alvarenga ${ }^{1}$ \\ orcid.org/0000-0001-9079-4956 \\ palvarenga66@gmail.com
}

\section{Zelma Freitas Soares ${ }^{1}$ \\ orcid.org/0000-0002-6741-3171 soareszelma@gmail.com}

\section{Paula Kleize Costa}

Sales $^{1}$

orcid.org/0000-0003-1069-3042 kleize@hotmail.com

\section{Nilton Correia dos Anjos Filho $^{1}$}

orcid.org/0000-0002-4901-2198 niltoncorreia_15@hotmail.com

Recebido em: 8/8/2018 Aprovado em: 23/10/2019. Publicado em: $x X \times x X .2020$.

\section{(c) (1)}

Artigo está licenciado sob forma de uma licença Creative Commons Atribuição 4.0 Internacional.
Resumo: Este estudo investigou o efeito mediador do conhecimento materno sobre o desenvolvimento infantil na relação entre escolaridade materna e indicadores desenvolvimentais no primeiro ano de vida. Participaram do estudo 28 mães de bebês com 11 meses de vida, selecionadas em duas Unidades Básicas de Saúde da cidade de Salvador/Brasil. O conhecimento materno sobre desenvolvimento foi avaliado com o Inventário do Conhecimento do Desenvolvimento Infantil (KIDI) e o desenvolvimento dos bebês foi medido pela Escala de Desenvolvimento do Comportamento da Criança (EDCC). As análises de regressão mostraram que o conhecimento materno sobre o desenvolvimento infantilé uma variável mediadora pura da relação entre a escolaridade materna e os indicadores desenvolvimentais do bebê. Discute-se o suporte fornecido pela educação formal para que os pais possam se beneficiar de informações sobre o desenvolvimento e recomenda-se o investimento na educação básica e na divulgação de informações sobre o desenvolvimento infantil em serviços públicos de saúde.

Palavras-chave: desenvolvimento infantil, escolaridade, mães, conhecimento, relações mãe-criança.

Abstract: This study investigated the mediating effect of maternal knowledge on child development in the relationship between maternal education level and developmental indicators in the first year. Twenty-eight mothers of 11-month-old infants, selected at two Basic Health Units in the city of Salvador/Brazil, participated in the study. Maternal developmental knowledge was assessed with the KIDI and infant development was measured by the EDCC. The regression analyses showed that maternal knowledge about child development is a pure mediating variable of the relationship between maternal schooling and developmental indicators of the baby. Support provided by formal education so that parents can benefit from developmental information is discussed, and investment in basic education and dissemination of information on child development in public health services is recommended.

Keywords: childhood development, educational status, mothers, awareness, motherchild relations.

Resumen: Este estudio investigo el efecto mediador del conocimiento materno sobre el desarrollo infantil en la relación entre escolaridad materna e indicadores del desarrollo en el primer año de vida. Participaron 28 madres de bebes con 11 meses de vida, seleccionadas en dos Unidades Básicas de Salud de Salvador/Brasil. El conocimiento materno sobre el desarrollo fue evaluado con el Inventario de Conocimiento del Desarrollo Infantil y el desarrollo de los bebes fue medido por la Escala de Desarrollo del Comportamiento del Niño. Los análisis de regresión mostraron que el conocimiento materno sobre el desarrollo infantil es una variable mediadora de la relación entre escolaridad materna y los indicadores de desarrollo del bebe. Se discute el soporte otorgado por la educación formal para que los padres puedan beneficiarse de informaciones sobre desarrollo y se recomienda inversión en educación básica y en divulgación de informaciones sobre desarrollo infantil en servicios públicos de salud. Palabras clave: desarrollo infantil, escolaridad, madres, conocimiento, relaciones madre-niño. 
O desenvolvimento humano envolve o surgimento, a modificação e o desaparecimento de comportamentos e habilidades (Pinto, Vilanova, \& Vieira, 1997). Ao longo do primeiro ano de vida, as habilidades adquiridas darão suporte para futuras aquisições nos diferentes domínios do desenvolvimento da criança (Pereira, Saccani, \& Valentini, 2016). No domínio motor, por exemplo, o comportamento evolui de padrões reflexos observados logo após o nascimento aos movimentos voluntários, que ao final do primeiro ano incluem a manipulação eficiente de objetos, erguer-se para ficar em pé e andar de forma coordenada com apoio (Bayley, 2006; Shaffer \& Kipp, 2012). Na esfera cognitiva, as mudanças mais marcantes ocorrem a partir dos oito meses quando a criança mostra capacidade de lembrar de brinquedos que foram escondidos e procurar por eles. No que se refere à linguagem, é também nessa faixa etária que ganhos expressivos importantes aparecem, como a vocalização de sons nasais e posteriormente sons de vogais e, mais tarde, em torno dos 11 meses, a capacidade de combinar duas sílabas, usar gestos para comunicar os seus desejos e reagir ao ouvir o seu próprio nome (Bayley, 2006; Safadi et al., 2016). Quanto ao desenvolvimento socioemocional, em torno do terceiro mês a criança já expressa notável interesse pelo rosto e voz humana e aos 11 meses de vida, imita sons, palavras ou ações do cuidador durante a interação (Bayley, 2006; Pinto et al., 1997).

O ritmo da aquisição dessas habilidades e o seu refinamento dependem da interação de fatores biológicos (Roberts, McCary, Shinkareva, \& Bailey, 2016; Saccani, Valentini, Pereira, Muller, \& Gabbard, 2013), e socioambientais (Koutra et al., 2013; Morais, Carvalho, \& Magalhães, 2016). Entre os fatores biológicos que podem afetar o desenvolvimento no primeiro ano de vida, estão o baixo escore no Apgar, a prematuridade, o baixo peso ao nascer e a baixa estatura (Datar \& Jacknowitz, 2009; Saccani et al., 2013). Quanto aos fatores socioambientais, tanto fatores sociodemográficos quanto aspectos relacionados à parentalidade, como as crenças e as práticas de cuidado e de socialização dos pais, têm recebido destaque (Marjanovič-Umek \& Fekonja-Peklaj, 2017; Mermelshtine \& Barnes, 2016; Scarzello, Arace, \& Prino, 2016; Wacharasin, Barnard, \& Spieker, 2003). Entre os aspectos sociodemográficos, a escolaridade e a renda parecem desempenhar um papel importante no desenvolvimento infantil. Bebês de familias que possuem menor poder aquisitivo e cujos pais têm menores níveis de escolaridade tendem a apresentar atrasos no desenvolvimento cognitivo e motor ao longo do primeiro ano (Clearfield, Stanger, \& Jenne, 2015; Koutra et al., 2013; Mermelshtine \& Barnes, 2016).

É possivel que esses fatores sociodemográficos atuem sobre variáveis ligadas à parentalidade que, por sua vez, afetam o desenvolvimento da criança. Alvarenga, Oliveira e Lins (2018) afirmam que tanto os estudos internacionais quanto as pesquisas realizadas no Brasil indicam dificuldades nas tarefas ligadas à parentalidade em familias socialmente vulneráveis, incluindo menor conhecimento sobre o desenvolvimento infantil, padrões de responsividade inadequadose práticas educativas parentais menos eficazes. Para os autores, a compreensão dessas relações passa pela escassez de recursos, a desorganização e a falta de estrutura dos bairros pobres e comunidades onde vivem familias socialmente vulneráveis, a rotina doméstica, muitas vezes, extenuante, devido ao elevado número de crianças demandando atenção e cuidados e a falta de acesso à educação formal de qualidade. Alvarenga et al. (2018) discutem o fato de que populações que não têm acesso à educação de qualidade encontram-se em desvantagem no que se refere às habilidades que dispõem para compreender e resolver problemas, incluindo questões domésticas e familiares. Nesse cenário, o nivel de instrução pode, por exemplo, restringir ou ampliar o conhecimento que os pais terão a respeito do desenvolvimento infantil.

Vários estudos confirmam o impacto do nível de escolaridade (Al-Maadadi \& Ikhlef, 2015; Cruz, Cavalcante, \& Pedroso, 2014; Marjanovič-Umek \& Fekonja-Peklaj, 2017; Ribas Jr., Moura, \& Bornstein, 
2003; Rowe, Denmark, Jones, \& Stapleton, 2016; Yue et al., 2017) e do status socioeconômico (Ribas Jr. et al., 2003; Rowe et al., 2016; Yue et al., 2017) sobre o conhecimento dos pais a respeito do desenvolvimento infantil. Estudos envolvendo mães com baixa escolaridade e baixa renda constataram pouco conhecimento sobre o desenvolvimento infantil por parte das mesmas. A maioria das participantes não sabia que a visão, as vocalizações, o sorriso social e o desenvolvimento do cérebro ocorrem desde os primeiros meses de vida ou que elas deveriam falar com seus filhos para estimular o seu desenvolvimento, por exemplo (Ertem et al., 2007; Rehman, Kazmi, \& Munir, 2016). No Brasil, Ribas Jr. et al. (2003) analisaram as relações entre o status socioeconômico e o conhecimento das mães acerca do desenvolvimento infantil em uma amostra de 64 mães primiparas. O nível de educação da mãe foi o melhor preditor do conhecimento materno em relação ao desenvolvimento infantil.

A idade da criança é apontada por alguns estudos como fator que também influencia o grau de conhecimento dos pais em relação ao desenvolvimento infantil (Al-Maadadi \& Ikhlef, 2015; Scarzello etal., 2016). Por exemplo, quanto mais velha a criança, mais os conhecimentos apresentados pelo cuidador. Isso indica que não é só o cuidador que influencia o desenvolvimento do bebê, mas que as pistas emitidas pelos comportamentos da criança contribuem para regular as atitudes do cuidador (Brummelte \& Galea, 2016; Moura et al., 2004). Além disso, as oportunidades de observar o desenvolvimento infantil, no caso de pais com mais de um filho, apresentam relações com o grau de conhecimento sobre o desenvolvimento infantil (Cruz et al., 2014; Scarzello et al., 2016).

O conhecimento que os pais possuem a respeito de como uma criança se desenvolve e de práticas que podem facilitar ou prejudicar esse processo é considerado um aspecto da cognição social adulta que está relacionada à compreensão dos processos de desenvolvimento infantil, das habilidades de cuidar e de educar as crianças e dos marcos do seu desenvolvimento (Dichtelmiller, Meisels, Plunkett, Bozynski, \& Mangelsdorf, 1992). Essa variável tem sido avaliada com diferentes estratégias metodológicas. 0
Knowledge of Infant Development Inventory (KIDI) inclui conhecimentos sobre diferentes áreas do desenvolvimento da criança, desde o nascimento até os dois anos de idade, divididos em quatro subescalas: a) Normas e marcos; b) Principios; c) Parentalidade; e d) Saúde e segurança (MacPhee, 1983: Scarzello et al., 2016). O Caregiver Knowledge of Child Development Inventory (CKCDI) avalia duas dimensões do conhecimento do cuidador: a) aparecimento de habilidades básicas do desenvolvimento; e b) idades apropriadas para fornecer oportunidades básicas para estimular o desenvolvimento infantil (Ertem et al., 2007; Rehman et al., 2016). Outro instrumento que avalia o conhecimento sobre o desenvolvimento infantil é o Catalog of Previous Experience with Infants (COPE), que mede a exposição prévia do cuidador a informações relacionadas à infância e às práticas de cuidados adotadas (Al-Maadadi \& Ikhlef, 2015; MacPhee, 1983). A análise dos três instrumentos indica que o conhecimento do cuidador em relação aos marcos do desenvolvimento infantil e as práticas de cuidados são as dimensões mais frequentemente investigadas.

Muitas investigações oferecem evidências de que o conhecimento dos pais sobre o desenvolvimento infantil afeta resultados desenvolvimentais da criança. Estudos realizados nas décadas de 1980 e 1990 constataram associações positivas entre o conhecimento do cuidador e as habilidades cognitivas, motoras e de linguagem da criança (Dichtelmiller et al., 1992; Stevens, 1984). Resultados semelhantes foram obtidos em estudos mais recentes. Por exemplo, Rowe et al. (2016) investigaram se o conhecimento dos pais aos nove meses de vida da criança explicaria as habilidades de linguagem das crianças de diferentes status socioeconômicos aos 48 meses. Foram encontradas correlações positivas entre o conhecimento dos pais e as habilidades de linguagem das crianças. Análises multivariadas testaram se o grau de conhecimento dos pais em relação ao desenvolvimento infantil mediaria a relação entre o nivel de educação dos pais e as habilidades de linguagem da criança. Foram encontrados tamanhos de efeito fracos, porém significativos, indicando o papel mediador dessa variável. 
As relações entre o conhecimento dos cuidadores sobre o desenvolvimento infantil e os resultados desenvolvimentais da criança na primeira infância foram também investigadas por Yue et al. (2017). O estudo foi realizado na China envolvendo 648 bebês na faixa etária de seis a 24 meses. Os resultados da análise multivariada mostraram que o maior conhecimento do cuidador acerca do desenvolvimento infantil esteve positivamente relacionado ao desenvolvimento socioemocional e de linguagem das crianças. No Brasil, um estudo transversal realizado com 49 familias constatou, a partir da análise de regressão linear simples, associação significativa entre o conhecimento dos cuidadores e o desenvolvimento motor da criança nos três momentos avaliados, aos oito, 10 e 12 meses (Pereira et al., 2016).

Diferentes processos podem explicar a relação entre o conhecimento dos pais sobre o desenvolvimento infantil e os resultados desenvolvimentais observados nos filhos. Uma das hipóteses explicativas prevê que a maior consciência e compreensão sobre o processo de desenvolvimento torne os pais mais sensiveis e responsivos ao comportamento dos filhos, o que, por sua vez, afetaria o desenvolvimento da criança. Nesse sentido, pouco conhecimento materno em relação às capacidades cognitivas da criança pode se refletir em pouca oferta de oportunidades capazes de estimular esse domínio (Donovan, Taylor, \& Leavitt, 2007; Scarzello et al., 2016; Wacharasin et al., 2003), assim como pode influenciar os tipos de brincadeira em que os pais se engajam com a criança (Marjanovič-Umek \& Fekonja-Peklaj, 2017; McMillin et al., 2015). Por outro lado, uma investigação recente não encontrou evidências a favor dessa hipótese. Trata-se de uma revisão sistemática da literatura que examinou a associação entre o conhecimento dos pais a respeito do desenvolvimento infantil e os estilos parentais (September, Rich, \& Roman, 2017). Foram incluidos oito estudos transversais e longitudinais, publicados na língua inglesa no periodo de 2003 a 2014. Os autores destacam a escassez de estudos envolvendo essas variáveis e apontam para o fato de que os estudos revisados não têm resultados consistentes sobre a relação entre o conhecimento do desenvolvimento infantil e a parentalidade.

Os estudos revisados pelos autores do presente estudo mostram que o grau de conhecimento dos pais sobre o desenvolvimento infantil está associado a fatores relacionados às condições de vida, principalmente no que diz respeito à escolaridade e renda (Al-Maadadi \& Ikhlef, 2015; September et al., 2017). O fato de a idade da criança interferir no conhecimento dos pais acerca do desenvolvimento infantil, por sua vez, ratifica a importância de estudos com bebês menores (Al-Maadadi \& Ikhlef, 2015; Scarzello et al., 2016). Muitas investigações oferecem evidências da relação entre essa variável e diferentes domínios do desenvolvimento infantil (Dichtelmiller et al., 1992; Rowe et al., 2016; Yue et al., 2017), contudo poucos estudos examinaram essa relação em amostras brasileiras (Pereira et al., 2016). Soma-se a isso o fato de que nenhum estudo realizado no Brasil considerou o papel mediador do conhecimento sobre o desenvolvimento infantil na relação entre escolaridade dos pais e resultados desenvolvimentais dos filhos. Assim, o presente estudo investigou o efeito mediador do conhecimento materno sobre o desenvolvimento infantil na relação entre a escolaridade materna e indicadores desenvolvimentais nos bebês aos 11 meses de vida. A hipótese que norteou essa investigação foi a de que a escolaridade materna seria um preditor significativo da frequência de indicadores desenvolvimentais aos 11 meses de vida da criança, sendo essa relação mediada pelo conhecimento sobre o desenvolvimento infantil.

\section{Método}

\section{Participantes}

Participaram do estudo 28 diades mãe-bebê, recrutadas junto a Unidades de Saúde da Familia de duas comunidades socioeconomicamente vulneráveis na cidade de Salvador, Brasil. Essa amostra foi selecionada para um estudo longitudinal mais amplo que avaliou os efeitos de uma intervenção para aumentar o nível de responsividade materna e favorecer o desenvolvimento da criança. As díades da amostra desta 
investigação fizeram parte do grupo controle do estudo referido. Os bebês (18 do sexo masculino e 10 do sexo feminino) tinham onze meses de vida no momento da coleta de dados, nasceram a termo e não apresentavam enfermidades crônicas físicas ou mentais. As mães tinham, aproximadamente, 27 anos de idade $(M=27,53 ; D P=6,03)$, estudaram, em média, 10,79 anos ( $M=10,79 ; D P=2,93)$, o que corresponde ao ensino médio incompleto, e 60,7\% delas não trabalhavam fora de casa. Os pais dos bebês estudaram, aproximadamente, 11 anos $(M=11,16 ; D P=2,58)$ e $74,1 \%$ deles exerciam alguma atividade remunerada fora de casa. A maior parte das mães vivia com o pai do bebê (82,1\%) e não apresentava problemas de saúde fisica $(85,7 \%)$. As quatro mães que relataram problemas de saúde fisica mencionaram anemia, diabetes, hipertensão, anemia falciforme ou rinite. A renda familiar foi de aproximadamente dois salários mínimos em reais ( $M=1.750,21 ; D P=973.51)$, conforme vigência no período da coleta de dados. Mais detalhes sobre as caracteristicas sociodemográficas podem ser consultadas na Tabela 1.

TABELA 1 - Caracteristicas Sociodemográficas das Participantes

\begin{tabular}{|c|c|c|c|c|}
\hline \multirow{2}{*}{ Variáveis } & \multirow{2}{*}{ Categorias } & \multicolumn{3}{|c|}{ Valores } \\
\hline & & $n$ & Média / Frequência & $D P$ \\
\hline \multicolumn{5}{|c|}{ Mãe } \\
\hline Idade (em anos) & & & 27.53 & 6,03 \\
\hline Escolaridade (em anos) & & & 10,79 & 2,93 \\
\hline \multirow{3}{*}{ Ocupação } & Trabalham fora & 11 & 39.3 & \\
\hline & & & & \\
\hline & Não trabalham fora & 17 & 60,7 & \\
\hline \multirow{3}{*}{ Problemas de saúde } & Sim & 4 & 14.3 & \\
\hline & & & & \\
\hline & Não & 24 & 85,7 & \\
\hline
\end{tabular}

Pai

\begin{tabular}{llcc}
\hline Escolaridade (em anos) & & 11,16 & 2,58 \\
Ocupação & Trabalham fora & 20 & 74,1 \\
& Não trabalham fora & 7 & 25,9 \\
\hline \multicolumn{3}{c}{ Bebê } \\
\hline \multirow{2}{*}{ Sexo } & Masculino & 18 & 64,3 \\
& Feminino & 10 & 35,7 \\
\hline
\end{tabular}

Familia

\begin{tabular}{lcccc}
\hline Quantidade de filhos além do bebê & & & 0,75 & 1,04 \\
Coabitação parental & Sim & 23 & 82,1 & 17,9 \\
& Não & 5 & 35,89 & 30,62 \\
Tempo da união conjugal (em meses) & & $1,740,21$ & 973,51 \\
Renda familiar (em reais) & & & \\
\hline
\end{tabular}

Nota. $N=28$.

aUma participante desconhecia a ocupação do pai do bebê 


\section{Instrumentos}

Ficha de dados sociodemográficos. Foi confeccionada especialmente para este estudo, com o objetivo de investigar dados como sexo do bebê, idade da mãe, escolaridade e profissão dos genitores, renda familiar e existência de outros filhos.

Inventário do conhecimento do desenvolvimento infantil - KIDI. Foi utilizada a versão em português do KIDI, traduzida e adaptada por Ribas Jr., Moura, Gomes e Soares (2000). O KIDI possui 75 questões, 48 das quais solicitam que 0 respondente escolha uma entre três respostas ("concordo", "discordo", ou "não estou certo") para uma série de declarações. Outras 20 questões pedem que o respondente opte poruma entre quatro respostas ("concordo", "mais jovem", "mais velha", "não tenho certeza") relacionadas às afirmações acerca de quando uma criança normalmente adquire uma determinada competência. Finalmente, sete questões solicitam que o respondente selecione uma entre cinco respostas. Os autores da versão adaptada recomendam a utilização do escore total no KIDI definido como a proporção total de itens respondidos corretamente (número de respostas corretas dividido pelo total de respostas). Adicionalmente, o KIDI pode fornecer resultados em quatro áreas ou subescalas distintas: (1) Normas; (2) Principios; (3) Parentalidade; e (4) Saúde e Segurança. No estudo de Silva, Vieira, Moura, e Ribas Jr. (2005) foi obtido um alfa de

\section{Cronbach de 0,74.}

\section{Escala de desenvolvimento do comportamento} da criança - EDCC. Desenvolvida no Brasil por Pinto et al. (1997), trata-se de um instrumento que, através de uma interação lúdica com a criança, permite a avaliação do desenvolvimento do comportamento motor e do comportamento atividade de crianças de um a 11 meses de idade. Os comportamentos motores, que envolvem respostas como levar a mão à boca ou engatinhar, são subdivididos quanto ao eixo somático e à estimulação. No que se refere ao eixo somático, os comportamentos motores pertencem à categoria axial, que corresponde ao eixo longitudinal crânio-caudal, ou à categoria apendicular, que contempla movimentos com os membros inferiores e superiores. Quanto à estimulação, esses comportamentos são subdivididos em estimulado ou espontâneo. Os comportamentos atividade, aqueles que se referem às respostas em busca de interação social, como emitir sons vocálicos ou virar-se quando chamado pelo nome, são subdivididos em dois tipos: a) comunicativos, cuja exteriorização indica uma determinação de contato e comunicação na interação da criança com outra pessoa. Exemplos de comportamentos comunicativos aos 11 meses são combinar duas silabas diferentes em jogo silábico e usar intencionalmente palavra com significado; e b) não comunicativos, quando a determinação de contato ou comunicação não ocorre. Exemplos de comportamentos não comunicativos aos 11 meses são dar alguns passos sem apoio e rabiscar. Nesse instrumento, os comportamentos são também agrupados em função da probabilidade de ocorrência, a cada mês de idade e para cada sexo, de modo que são classificados em três categorias: a) aparecimento, agrupa comportamentos que não são típicos daquela fase do desenvolvimento, mas que podem estar presentes, pois foram observados em, pelo menos, uma criança daquela faixa etária; b) normalização, reúne comportamentos apresentados por cerca de 50\% das crianças naquela faixa etária; e c) estabilização, agrupa comportamentos presentes em aproximadamente 90\% das crianças daquela faixa etária. Com essa classificação é possivel observar as idades, em meses, nas quais cada comportamento tende a aparecer, a ser comum (normalizar) e a ser altamente frequente (estabilizar-se), permitindo uma indicação do ritmo de desenvolvimento e possibilitando uma estimativa acerca de atrasos ou aceleração do desenvolvimento. A soma dos comportamentos apresentados em cada uma das três categorias foi considerada para o escore na referida categoria. A partir desse escore foram geradas proporções nas categorias aparecimento, normalização e estabilização, dividindo-se o total de comportamentos observados em cada categoria pelo número total de itens da escala na referida categoria para os meninos e para as meninas. $O$ mesmo procedimento de cálculo de proporções foi utilizado para as categorias de comportamentos comunicativos e não comunicativos. Essas proporções foram utilizadas como escores 
ponderados em todas as análises realizadas.

\section{Procedimentos}

Coleta de dados. Após aprovação pelo Comitê de Ética em Pesquisa da Faculdade de Medicina da Bahia, da Universidade Federal da Bahia (Parecer n. ${ }^{\circ}$ 396.333), familias de gestantes cadastradas em Unidades de Saúde da Familia de duas comunidades socioeconomicamente vulneráveis, em Salvador/Brasil, foram convidadas para participar de um estudo longitudinal. Os dados do presente estudo correspondem à etapa do pós-teste realizada com o grupo controle após o período de tratamento com o grupo intervenção no estudo mencionado. As mães que concordaram com a participação receberam uma visita domiciliar, quando o bebê tinha onze meses de vida, na qual assinaram um Termo de Consentimento Livre e Esclarecido (TCLE), forneceram informações sociodemográficas da familia e responderam ao KIDI em forma de entrevista. O pesquisador lia os itens e as alternativas de resposta e a mãe informava a resposta escolhida, com auxilio de um cartão de respostas. O desenvolvimento dos bebês foi avaliado pela EDCC durante a mesma visita. Os pesquisadores responsáveis por essa avaliação foram treinados para a aplicação dos instrumentos e realizaram testes piloto antes de iniciar a coleta de dados. A pesquisa envolveu riscos mínimos aos participantes. A avaliação do desenvolvimento do bebê e do conhecimento da mãe sobre o desenvolvimento poderiam causar às participantes algum tipo de constrangimento ou preocupação com relação ao desempenho dos bebês no teste ou algum desconforto para os bebês. No caso da ocorrência de quaisquer dessas situações o pesquisador deveria interromper a coleta, esclarecer as implicações dos resultados do bebê no teste e, caso necessário, encaminhar a diade para avaliação e acompanhamento psicológico. Contudo, nenhuma dessas situações ocorreu.

Análise dos dados. Inicialmente foram realizadas análises descritivas, incluindo mínimo, máximo, média, desvio padrão e intervalo de confiança (95\%), do escore total e das quatro subescalas do KIDI e da proporção do escore total da EDCC, bem como das categorias específicas da EDCC. Em seguida, foram utilizados testes de correlação bivariada e de regressão linear múltipla. O teste de Pearson foi adotado devido à distribuição simétrica verificada na maioria das variáveis. As correlações foram classificadas com base na intensidade do relacionamento entre as variáveis (fraca: maior que zero a 0,4; moderada: de 0,41 a 0,7; forte: maiores do que 0,71) (Dancey \& Reidy, 2006). Dois modelos de regressão linear simples foram testados e analisados conjuntamente para determinar o possivel efeito mediador do conhecimento materno sobre o desenvolvimento infantil sobre a relação entre a escolaridade e os resultados desenvolvimentais da criança (Abbad \& Torres, 2002; Hayes, 2018; Tabachnick \& Fidel, 2013). O ponto de corte utilizado para a avaliação da significância de todos os valores de alpha foi 0,05.

\section{Resultados}

As análises de estatística descritiva, utilizadas para caracterização da amostra, indicaram que a média do escore no KIDI (conhecimento materno sobre o desenvolvimento infantil) foi de 0,61 ( $D P=0,11 ; / C=0,56-0,65)$. Os escores nessa escala variaram entre 0,36 e 0,77. Também foi calculada a proporção de respostas corretas em cada uma das subescalas do KIDI. O domínio do desenvolvimento da criança com maior número de respostas corretas das mães foi Marcos ou Normas, com média de itens respondidos corretamente igual a 17,39 $(D P=4,03$; IC=15,83-18,96), seguido pelo domínio Princípios $(M=10,36 ; D P=2,53 ; / C=9,38-11,34)$. A média de itens respondidos de forma correta no domínio Parentalidade foi igual a $9(D P=2,21 ; I C=8,14-9,86)$ e no campo Saúde e Segurança foi equivalente a 8,82 ( $D P=1,47 ; / C=8,25-9,39$ ).

Quanto aos indicadores do desenvolvimento infantil, as análises de estatística descritiva revelaram que o escore proporcional médio da EDCC foi de $0,71(D P=0,12 ; / C=0,66-0,76)$. As proporções de escore total nesse instrumento variaram entre 0,39 e 0,91. Para avaliar o desenvolvimento 
da criança, foram efetuadas também análises de estatística descritiva dos comportamentos atividade da criança, assim como da probabilidade de ocorrência de tais comportamentos. Quanto aos comportamentos atividade, a proporção média de comportamentos comunicativos foi de 0,67 $(D P=0,15 ; / C=0,61-0,73)$ e de comportamentos não comunicativos foi igual a $0,71(D P=0,17 ; / C=0,65$ 0,78). Em relação à probabilidade de ocorrência dos comportamentos, a proporção média dos comportamentos em estabilização foi de 0,86 $(D P=0,18 ; / C=0,79-0,93)$, em normalização foi igual a $0,80(D P=0,15 ; / C=0,74-0,85)$ e em aparecimento foi de $0,63(D P=0,17 ; / C=0,56-0,70)$.

\section{Relações entre as variáveis sociodemográficas e o conhecimento materno sobre o desenvolvimento infantil}

O teste de Pearson revelou que o escore total do KIDI teve correlação positiva moderada com a escolaridade da mãe $(r=0,54 ; p<0,01)$.
As correlações entre as demais variáveis sociodemográficas (idade, escolaridade do pai e número de filhos) e o escore total da mãe no KIDI não foram significativas. Os resultados obtidos com base no teste de Pearson também mostraram correlações entre as subescalas do KIDI e as variáveis sociodemográficas. A escolaridade da mãe correlacionou-se positivamente em nivel moderado com o conhecimento da mãe sobre Marcos ou Normas $(r=0,52 ; p<0,01)$, Principios $(r=0,54 ; p<0,01)$ e Parentalidade $(r=0,35 ; p<0,05)$. A quantidade de filhos correlacionou-se negativamente em nivel fraco com o conhecimento da mãe sobre Princípios $(r=-0,33 ; p<0,05)$ e Saúde e Segurança $(r=-0,32 ; p<0,05)$. As demais relações entre as variáveis sociodemográficas e o conhecimento materno em cada área do desenvolvimento do bebê não foram significativas. A Tabela 2 resume esses resultados.

TABELA 2 - Correlações entre as Variáveis Sociodemográficas, o Conhecimento Materno sobre o Desenvolvimento Infantil e os Indicadores Desenvolvimentais da Criança

\begin{tabular}{|c|c|c|c|c|c|c|c|c|c|c|c|c|c|c|c|c|}
\hline Variáveis & 1 & 2 & 3 & 4 & 5 & 6 & 7 & 8 & 9 & 10 & 11 & 12 & 13 & 14 & 15 & 16 \\
\hline 1. Idadeda mãe & - & & & & & & & & & & & & & & & \\
\hline $\begin{array}{l}\text { 2. Escolaridade da } \\
\text { mãe }\end{array}$ & 0,20 & - & & & & & & & & & & & & & & \\
\hline 3. Escolaridade do pai & 0,24 & 0,21 & - & & & & & & & & & & & & & \\
\hline 4. Número de irmãos & $0,46^{* *}$ & $-0.35^{*}$ & 0 & - & & & & & & & & & & & & \\
\hline 5. Renda & $-0,36^{*}$ & 0,10 & 0,17 & $-0,26$ & - & & & & & & & & & & & \\
\hline 6. Marcos $^{a}$ & 0,00 & $0.52^{* *}$ & 0,02 & $-0,07$ & 0,18 & - & & & & & & & & & & \\
\hline 7. Principios ${ }^{a}$ & $-0,24$ & $0.54^{* *}$ & $-0,02$ & $-0,33^{*}$ & 0,20 & $0,64^{* *}$ & - & & & & & & & & & \\
\hline 8. Parentalidade ${ }^{a}$ & $-0,17$ & $0.35^{*}$ & 0,03 & 0,03 & 0,24 & $0,64^{* *}$ & $0,63^{* *}$ & - & & & & & & & & \\
\hline 9. Saúde e segurança ${ }^{a}$ & $-0,09$ & 0,23 & 0 & $-0,32^{*}$ & 0,27 & 0,29 & 0,25 & 0,30 & - & & & & & & & \\
\hline 10. KIDI & $-0,12$ & $0.54^{* *}$ & 0,02 & $-0,17$ & 0,27 & $0,90^{* *}$ & $0,83^{* *}$ & $0,82^{* *}$ & $0,48^{* *}$ & - & & & & & & \\
\hline 11. Comunicativos ${ }^{b}$ & $-0,08$ & 0.30 & 0,06 & $-0,40^{*}$ & 0,23 & 0,29 & $0,46^{* *}$ & 0,17 & $0,48^{* *}$ & $0,41^{*}$ & - & & & & & \\
\hline 12. Não comunicativos ${ }^{\text {b }}$ & $-0,08$ & 0,20 & 0,19 & 0,00 & $-0,02$ & $0.37^{*}$ & 0.31 & $0,44^{* *}$ & $0,32^{*}$ & $0,46^{* *}$ & 0,08 & - & & & & \\
\hline 13. Estabilizaçãob & 0,10 & 0,05 & 0,05 & $-0,06$ & $-0,30$ & 0,11 & 0,14 & 0,01 & $0,36^{*}$ & 0,18 & 0,20 & $0,42^{*}$ & - & & & \\
\hline 14. Normalizaçãob & $-0,34^{*}$ & 0,22 & 0,07 & $-0,38^{*}$ & 0,19 & $0,31^{*}$ & $0,46^{* *}$ & $0,37^{*}$ & $0,54^{* *}$ & $0,49^{* *}$ & $0,62^{* *}$ & $0,31^{*}$ & 0,13 & - & & \\
\hline 15. Aparecimento ${ }^{b}$ & 0,06 & $0.34^{*}$ & 0,26 & $-0,17$ & 0,18 & $0.37^{*}$ & $0,46^{* *}$ & $0.35^{*}$ & $0,33^{*}$ & $0,48^{* *}$ & $0,65^{\star *}$ & $0,64^{* *}$ & 0,18 & $0,33^{*}$ & - & \\
\hline 16. EDCC & $-0,06$ & $0,36^{*}$ & 0,24 & $-0,29$ & 0,16 & $0,42^{*}$ & $0.54^{* *}$ & $0,39^{*}$ & $0.51^{* \star}$ & $0.58^{* *}$ & $0,74^{* *}$ & $0,70^{* *}$ & $0,38^{*}$ & $0,61^{* *}$ & $0,92^{* *}$ & - \\
\hline
\end{tabular}

Nota. $N=28 . \mathrm{KIDI}=$ Knowledge of Infant DevelopmentInventory; EDCC = Escala de Desenvolvimento do

Comportamento da Criança.

a Subescalas do KIDI. ' $\mathrm{C}$ ategorias especificas geradas pela EDCC.

${ }^{*} p<0.05{ }^{* *} p<0.01$ 
Relações entre as variáveis sociodemográficas e o desenvolvimento infantil

O nivel de escolaridade materna esteve correlacionado positivamente em nivel fraco com o escore proporcional total da criança na $\operatorname{EDCC}(r=0,36 ; p<0,05)$. Quanto aos escores nas categorias especificas geradas pela EDCC, a idade da mãe teve correlação negativa fraca com a proporção de comportamentos em normalização da criança $(r=-0,34 ; p<0,05)$. A quantidade de filhos correlacionou-se negativamente em nivel fraco com a proporção de comportamentos comunicativos $(r=-0,40 ; p<0,05)$ e em normalização da criança $(r=-0,38 ; p<0,05)$. A escolaridade da mãe teve correlação positiva fraca com a proporção de comportamentos em aparecimento da criança $(r=0,34 ; p<0,05)$. As demais relações entre o desenvolvimento infantil e as variáveis sociodemográficas não foram significativas. A Tabela 2 apresenta esses resultados.

\section{Relações entre o conhecimento materno sobre o desenvolvimento infantil e o desenvolvimento da criança}

O teste de Pearson evidenciou correlação positiva moderada entre o escore total do KIDI e o escore proporcional total na EDCC $(r=0,58$; $p<0,01$ ). Os resultados também mostraram correlações positivas entre a proporção na EDCC e o conhecimento da mãe sobre cada domínio do desenvolvimento da criança. O escore proporcional total da criança na EDCC teve correlação positiva fraca com o conhecimento da mãe sobre Parentalidade $(r=0,39 ; p<0,05)$ e moderada com as subescalas Marcos ou Normas $(r=0,42 ; p<0,05)$, Principios $(r=0,54 ; p<0,01)$ e Saúde e Segurança $(r=0,51 ; p<0,01)$.

Verificou-se também relações entre o conhecimento da mãe acerca do desenvolvimento infantil (KIDI) e a proporção de comportamentos atividade da criança na EDCC. O escore total no KIDI teve correlação positiva moderada com a proporção de comportamentos comunicativos $(r=0,41 ; p<0,05)$ e não comunicativos da criança $(r=0,46 ; p<0,01)$. Houve também correlações entre os escores da mãe nas subescalas do
KIDI e a proporção de comportamentos atividade da criança. A proporção de comportamentos comunicativos da criança correlacionouse positivamente em nivel moderado com o conhecimento da mãe sobre Saúde e Segurança $(r=0,48 ; p<0,01)$ e Principios do Desenvolvimento $(r=0,46 ; p<0,01)$. A proporção de comportamentos não comunicativos correlacionou-se positivamente em nivel fraco com o total de respostas corretas da mãe sobre Marcos e Normas $(r=0,37 ; p<0,05)$ e Saúde e Segurança $(r=0,32 ; p<0,05)$ e em nivel moderado com Parentalidade $(r=0,44 ; p<0,01)$. As demais relações entre o conhecimento materno sobre o desenvolvimento da criança e o desempenho infantil não foram significativas.

Os resultados também mostraram relações entre o conhecimento da mãe sobre o desenvolvimento infantil e o ritmo de desenvolvimento. O escore total no KIDI teve correlação positiva moderada com a proporção de comportamentos em normalização $(r=0,49 ; p<0,01)$ e em aparecimento $(r=0,48 ; p<0,01)$. Também se constatou que o conhecimento materno em cada campo específico do desenvolvimento infantil relacionou-se com o ritmo do desenvolvimento da criança. A proporção de comportamentos em normalização da criança teve correlação positiva fraca com o total de respostas corretas da mãe sobre Marcos ou Normas $(r=0,31 ; p<0,05)$ e Parentalidade $(r=0,37 ; p<0,05)$ e moderada sobre Principios do Desenvolvimento $(r=0,46$; $p<0,01$ ). A proporção de comportamentos em aparecimento correlacionou-se positivamente em nivel fraco com o total de respostas corretas em Marcos ou Normas $(r=0,37 ; p<0,05)$, Parentalidade $(r=0,35 ; p<0,05)$ e Saúde e Segurança $(r=0,33$; $p<0,05)$ e em nível moderado com Princípios do Desenvolvimento $(r=0,46 ; p<0,01)$. Além disso, o conhecimento da mãe sobre Saúde e Segurança teve correlação positiva fraca com a proporção dos comportamentos apresentados pela criança em estabilização $(r=0,36 ; p<0,05)$ e em normalização, $(r=0,54 ; p<0,01)$. As demais relações entre o conhecimento da mãe sobre o desenvolvimento infantil e a probabilidade 
de ocorrência de comportamentos observada na criança não foram significativas. A Tabela 2 resume esses resultados.

\section{Mediação do conhecimento materno sobre o desenvolvimento infantil na relação entre escolaridade materna e indicadores desenvolvimentais do bebê}

A hipótese de mediação foi testada de acordo a definição de variável mediadora de Abbad e Torres (2002), com o modelo quatro de mediação simples de Hayes (2018), com a proposta de análise passo a passo dos caminhos de Vieira (2009) e com os critérios para análise dos valores beta estabelecidos por Tabachnick e Fidel (2013). Por definição, uma variável mediadora é aquela que, ao estar presente em uma equação de regressão, diminui a magnitude do relacionamento entre uma variável antecedente e uma variável dependente ou critério (Abbad \& Torres, 2002, p. 21). Tabachnick e Fidel (2013) propõem a análise dos valores beta para confirmar o efeito mediador de uma variável. No caso de uma variável mediadora pura, o beta associado à variável antecedente deixaria de ser significativo com a entrada da variável mediadora na equação. Já quando a mediação não é total, o beta da variável antecedente diminui com a entrada da variável mediadora, embora ainda se mantenha significativo.

Inicialmente, a correlação positiva moderada entre a escolaridade materna e o conhecimento materno sobre desenvolvimento infantil confirma a relação entre variável antecedente e variável mediadora $(r=0,54 ; p<0,01)$. Para verificar os demais pressupostos de mediação, inicialmente foram testados dois modelos de regressão linear simples. O primeiro incluiu somente a escolaridade materna como variável antecedente e o escore proporcional total na EDCC como variável critério. O segundo incluiu somente o conhecimento materno como variável antecedente e o escore proporcional total na EDCC como variável critério. Em seguida foi testado um terceiro modelo de regressão linear múltipla que incluiu simultaneamente a escolaridade e o conhecimento materno sobre desenvolvimento infantil, para verificar se a inserção dessa variável diminuiria a magnitude da relação entre a escolaridade materna e o escore total na EDCC.

O primeiro modelo de regressão confirmou o poder preditivo da escolaridade materna $(p=0,05)$ sobre o desenvolvimento infantil (escore na EDCC), explicando $10 \%$ da variância $\left(R^{2}=0,10\right)$ no escore global de desenvolvimento da criança $(F(1)=4,01 ; p=0,05)$. A cada desvio-padrão a mais na escolaridade materna, o escore proporcional total na EDCC aumentou em 0,36 desvios-padrão. O segundo modelo de regressão confirmou o poder preditivo do conhecimento materno sobre o desenvolvimento $(p=0,001)$ sobre o escore proporcional total na EDCC, explicando 31,2\% da variância $\left(R^{2}=0,312\right)$ no escore global de desenvolvimento da criança $(F(1)=13,27 ; p=0,001)$. A cada desvio-padrão a mais no escore do KIDI, o escore proporcional total na EDCC aumentou em 0.58 desvios-padrão. A correlação entre variável antecedente e variável mediadora e os resultados das duas regressões indicaram o potencial de mediação do conhecimento materno sobre desenvolvimento infantil.

No terceiro modelo de regressão, a introdução do conhecimento sobre o desenvolvimento infantil como preditor fez com que a escolaridade materna deixasse de explicar a variância nos escores proporcionais totais da EDCC (desenvolvimento infantil). Os resultados mostraram que o conhecimento materno sobre o desenvolvimento infantil $(p=0,01)$ explicou $35,2 \%$ da variância $\left(R^{2}=0,352\right)$ no escore global de desenvolvimento da criança $(F(2,25)=6,78 ; p=0,004)$. A cada desvio-padrão a mais no KIDI (conhecimento materno sobre desenvolvimento infantil), o escore proporcional total na EDCC aumentou em 0,54 desviospadrão. A escolaridade materna deixou de ser um preditor significativo $(p=0,42)$. Os resultados das três equações de regressão analisadas em conjunto, indicam que o conhecimento materno sobre o desenvolvimento infantil é uma variável mediadora pura da relação entre a escolaridade materna e os indicadores desenvolvimentais do 
bebê aos 11 meses. A Figura 1 mostra os valores de $B$ e $p$ da análise de mediação, que explicou $35,2 \%$ da variância $\left(R^{2}=0,352\right)$ no escore global de desenvolvimento da criança $(F(2,25)=6,78$; $\mathrm{p}=0,004)$. Os resultados da análise de mediação mostraram que a escolaridade materna não teve efeito direto significativo sobre o desenvolvimento infantil $(p=0,42)$, mas indireto, via conhecimento materno a respeito do desenvolvimento da criança. Especificamente, a escolaridade materna teve um efeito significativo sobre o $\mathrm{KIDI}(B=$ $0,02, p<0,01)$ que, por sua vez, teve um efeito significativo na $\operatorname{EDCC}(B=0,54, p=0,01)$. O intervalo de confiança para o efeito indireto, estimado por 10.000 bootstraps, foi inteiramente acima de zero ( $B=0,01, I C$ 95\% [0,001, 0,023]), indicando que houve um efeito indireto estatisticamente significativo da escolaridade materna sobre a EDCC, através do KIDI (Hayes, 2018).

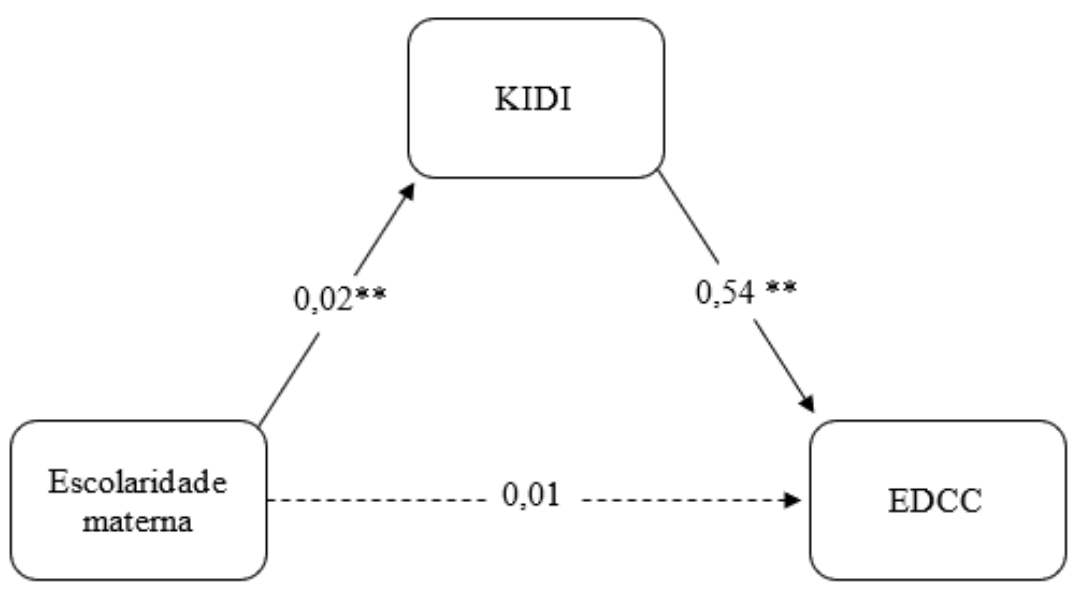

Figura 1 - Efeitos da escolaridade sobre o desenvolvimento infantil, mediados pelo conhecimento materno a respeito do desenvolvimento da criança. Linhas sólidas representam efeitos diretos. A linha tracejada representa o efeito indireto. Caminhos não significativos não são representados. EDCC = Escala de Desenvolvimento do Comportamento da Criança; KIDI = Inventário do Conhecimento do Desenvolvimento Infantil; ${ }^{* *} \mathrm{p} \leq 0,01$.

\section{Discussão}

As análises de regressão mostraram que o conhecimento materno sobre o desenvolvimento infantil é uma variável mediadora pura da relação entre a escolaridade materna e os indicadores desenvolvimentais do bebê. A hipótese sobre o efeito mediador do conhecimento sobre o desenvolvimento infantil já havia sido levantada na literatura (Rowe et al., 2016), e os resultados deste estudo confirmam de forma consistente o impacto dessa variável na relação analisada.

A escolarização formal constitui um meio fundamental de acesso para o desenvolvimento cognitivo, que amplia a capacidade de pensar e resolver problemas (Alvarenga et al., 2018). Ao mesmo tempo, o nivel de escolaridade está também relacionado à maior capacidade de compreender o desenvolvimento infantil e usufruir de informações a esse respeito (Bornstein, Hahn, Suwalsky, \& Haynes,
2003; Ribas Jr. et al., 2003). Assim, habilidades cognitivas satisfatórias dariam suporte para o melhor conhecimento sobre o desenvolvimento infantil, o que, por sua vez, contribuiria para a ampliação do repertório comportamental dos pais e, consequentemente, para melhores resultados desenvolvimentais nos filhos.

Em sintese, a confirmação do modelo de mediação indica que a escolaridade potencializa o uso que os pais podem fazer de informações sobre o desenvolvimento infantil, ao mesmo tempo em que corrobora a noção de que esse tipo de conhecimento é um fator crucial que favorece ou restringe o desenvolvimento da criança. Esse pressuposto foi corroborado também pelas inúmeras correlações verificadas entre os escores no KIDI e diferentes indicadores desenvolvimentais do bebê. Por exemplo, os conhecimentos das mães sobre marcos e sobre princípios do 
desenvolvimento da criança mostraram-se particularmente relevantes para os resultados da EDCC aos 11 meses, tanto no que se refere ao total de comportamentos observados, quanto no que diz respeito ao ritmo do desenvolvimento. De qualquer modo, o conhecimento sobre saúde e segurança também esteve correlacionado ao ritmo do desenvolvimento. É provável que mães que conheçam o potencial e os limites do comportamento do bebê em cada faixa etária e que tenham expectativas realistas sobre o que o bebê é capaz de fazer, ofereçam estimulação mais adequada durante a interação do que mães que não possuem esse tipo de conhecimento (Donovan et al., 2007; Marjanovič-Umek \& FekonjaPeklaj, 2017; McMillin et al., 2015; Scarzello et al., 2016; Wacharasin et al., 2003).

Além da confirmação da hipótese do estudo, as análises correlacionais exploratórias indicam a interdependência entre fatores associados às condições de vida, ao conhecimento materno sobre desenvolvimento infantil e aos resultados desenvolvimentais dos bebês. Além da escolaridade materna, a quantidade de irmãos correlacionou-se negativamente com a proporção de comportamentos comunicativos da criança. Essa relação merece ser melhor explorada em futuros estudos, na medida em que sugere que a presença de mais filhos pode reduzir a quantidade e a qualidade da estimulação que a criança recebe, o que poderia afetar o seu desenvolvimento.

Este estudo tem limitações. Não foi possivel avaliar o conhecimento do pai sobre o desenvolvimento infantil e o impacto dessa variável sobre o desenvolvimento da criança. Além disso, o tamanho reduzido da amostra pode ter diminuido o tamanho dos efeitos encontrados. Além do tamanho da amostra, o conhecimento e o desenvolvimento da criança poderiam ser avaliados com diferentes instrumentos e incluir outros informantes tais como o pai ou outros cuidadores. Nesse sentido, recomenda-se que novas investigações continuem explorando a hipótese de que a escolaridade do cuidador seria um preditor significativo da frequência de indicadores desenvolvimentais infantis, sendo essa relação mediada pelo conhecimento sobre o desenvolvimento, com novos delineamentos e instrumentos e com amostras maiores e mais variadas.

O presente estudo amplia as evidências a respeito do impacto da escolaridade materna sobre o desenvolvimento infantil, revelando a mediação do conhecimento sobre o desenvolvimento nessa relação. Os resultados indicam de forma consistente que a educação formal constitui um suporte fundamental para que os pais possam se beneficiar de informações sobre o desenvolvimento infantil, possivelmente transformando-as em práticas de cuidado e estimulação eficazes para favorecer o desenvolvimento dos filhos. Nesse sentido, recomenda-se fortemente o investimento na educação básica de qualidade e na divulgação de informações sobre o desenvolvimento infantil nos serviços públicos de saúde.

\section{Referências}

Abbad, G., \& Torres, C. V. (2002). Regressão múltipla stepwise e hierárquica em Psicologia Organizacional: aplicações, problemas e soluções. Estudos de Psicologia (Natal), 7(spe), 19-29. http://dx.doi. org/10.1590/S1413-294X2002000300004

Al-Maadadi, F., \& Ikhlef, A. (2015). What Mothers Know About Child Development and Parenting in Qatar: Parenting Cognitions and Practices. The Family Journal, 23(1), 65-73. https://doi. org/10.1177/1066480714555669

Alvarenga, P., Oliveira, J. M. de, \& Lins, T. C de S. (2018). Reflexões sobre a parentalidade no contexto de vulnerabilidade social no Brasil. In. L. F. Pêssoa, D. M. L. F. Mendes, \& M. L. S. Moura (Orgs.), Parentalidade: Diferentes perspectivas, evidências e experiências (pp.41-62). Curitiba: Appris. (DOI INEXISTENTE)

Bayley, N. (2006). Bayley Scales of Infant and Toddler Development: Administration Manual (3rd ed.). San Antonio, TX: The Psychological Corporation. (DOI

\section{INEXISTENTE)}

Bornstein, M. H., Hahn, C. S., Suwalsky, J. T. D., \& Haynes, O. M. (2003). Socioeconomic status, parenting, and child development: The Hollingshead four-factor index of social status and The Socioeconomic Index of Occupations. In. M. H. Bornstein, \& R. $\mathrm{H}$. Bradley (Orgs.), Socioeconomic status, parenting, and child development (pp. 29-81). Mahwah, New Jersey: Lawrence Erlbaum Associates. https://doi. org/10.4324/9781410607027 
Brummelte, S., \& Galea, L. A. M. (2016). Postpartum depression: Etiology, treatment and consequences for maternal care. Hormones and Behavior, 77. 153-166. https://doi.org/10.1016/j.yhbeh.2015.08.008

Clearfield, M. W., Stanger, S. B., \& Jenne, H. K. (2015). Socioeconomic status (SES) affects means-end behavior across the first year. Journal of Applied Developmental Psychology, 38, 22-28. https://doi. org/10.1016/j.appdev.2015.02.001

Cruz, E. J. S. da, Cavalcante, L. I. C., \& Pedroso, J. da S. (2014). Inventário de conhecimento do desenvolvimento infantil: estudo com mães de crianças em acolhimento institucional. Revista da SPAGESP, 15(1), 49-63. (DOI INEXISTENTE)

Dancey, C. P., \& Reidy, J. (2006). Estatistica sem Matemática para Psicologia. (3ed.). Porto Alegre: Artmed. (DOI INEXISTENTE)

Datar, A., \& Jacknowitz, A. (2009). Birth weight effects on children's mental, motor, and physical development: Evidence from twins data. Maternal and Child Health Journal, 13(6), 780-794. https://doi. org/10.1007/s10995-009-0461-6

Dichtelmiller, M., Meisels, S. J., Plunkett, J. W., Bozynski, M. E. A., \& Mangelsdorf, S. C. (1992). The Relationship of Parental Knowledge to the Development of Extremely Low Birth Weight Infants. Journal of Early Intervention, 16(3), 210-220. https://doi. org/10.1177/105381519201600302

Donovan, W., Taylor, N., \& Leavitt, L. (2007). Maternal Self-Efficacy, Knowledge of Infant Development, Sensory Sensitivity, and Maternal Response During Interaction. Developmental Psychology, 43(4), 865-876. https://doi.org/10.1037/0012-1649.43.4.865

Ertem, I. O., Atay, G., Dogan, D. G., Bayhan, A., Bingoler, B. E., Gok, C. G., ... Isikli, S. (2007). Mothers' knowledge of young child development in a developing country. Child: Care, Health and Development, 33(6), 728-737. https://doi.org/10.1111/j. 1365-2214.2007.00751.x

Hayes, A. F. (2018). Introduction to mediation, moderation, and conditional process analysis: A regression-based approach. 2nd. ed. New York: Guilford Press. (DOI INEXISTENTE)

Koutra, K., Chatzi, L., Bagkeris, M., Vassilaki, M., Bitsios, P., \& Kogevinas, M. (2013). Antenatal and postnatal maternal mental health as determinants of infant neurodevelopment at 18 months of age in a mother-child cohort (Rhea Study) in Crete, Greece. Social Psychiatry and Psychiatric Epidemiology, 48(8), 1335-1345. https://doi.org/10.1007/s00127-0120636-0

MacPhee, D. (1983). The nature of Parents' Experiences with and Knowledge about Infant Development. Paper Presented at the Biennial Meeting of the Society for Research in Child Development, 1-15. Recuperado de http://files.eric.ed.gov/fulltext/ED232788.pdf. (DOI INEXISTENTE)
Marjanovič-Umek, L., \& Fekonja-Peklaj, U. (2017). The Roles of Child Gender and Parental Knowledge of Child Development in Parent-Child Interactive Play. Sex Roles, 77(7-8), 496-509. https://doi.org/10.1007/ s11199-016-0734-7

McMillin, S. E., Hall, L., Bultas, M. W., Grafeman, S. E., Wilmott, J., Maxim, R., \& Zand, D. H. (2015). Knowledge of child development as a predictor of mother-child play interactions. Clinical Pediatrics, 54(11), 1117-1119. https://doi.org/10.1177/0009922815581763

Mermelshtine, R., \& Barnes, J. (2016). Maternal Responsive-didactic Caregiving in Play Interactions with 10-month-olds and Cognitive Development at 18months. Infant and Child Development, 25(3), 296-316. https://doi.org/10.1002/icd.1961

Morais, R. L. S., Carvalho, A. M., \& Magalhães, L. C. (2016). O contexto ambiental e o desenvolvimento na primeira infância: estudos brasileiros. Journal of Physical Education, 27(1), 1-14. https://doi.org/10.4025/ jphyseduc.v27i1.2714

Moura, M. L. S. de, Ribas Jr., R. de C., Piccinini, C. A., Bastos, A. C. de S., Magalhães, C. M. C., Vieira, M. L., ... Silva, A. K. da. (2004). Conhecimento sobre desenvolvimento infantil em mães primiparas de diferentes centros urbanos do Brasil. Estudos de Psicologia, 9(3), 421-429. https://doi.org/10.1590/ S1413-294X2004000300004

Pereira, K. R. G., Saccani, R., \& Valentini, N. C. (2016). Cognição e ambiente são preditores do desenvolvimento motor de bebês ao longo do tempo. Fisioterapia e Pesquisa, 23(1), 59-67. https://doi. org/10.1590/1809-2950/14685223012016

Pinto, E. B., Vilanova, L. C. P., \& Vieira, R. M. (1997). O desenvolvimento do comportamento da criança no primeiro ano de vida: padronização de uma escala para a avaliação e o acompanhamento. São Paulo: Casa do Psicólogo: FAPESP. (DOI INEXISTENTE)

Rehman, A. U., Kazmi, S., \& Munir, F. (2016). Mothers' Knowledge about Child Development. Pediatr J. 40(3), 176-81. (DOI INEXISTENTE)

Ribas Jr., R. D. C., Moura, M. L. S. de, Gomes, A. A. N., \& Soares, I. D. (2000). Adaptação brasileira do Inventário de Conhecimento sobre o Desenvolvimento Infantil de David Macphee [Resumo]. In Sociedade Brasileira de Psicologia do Desenvolvimento (Org.), Anais - III Congresso Brasileiro de Psicologia do Desenvolvimento (p. 183). Niterói, RJ: Autor. (DOI INEXISTENTE)

Ribas Jr., R. D. C., Moura, M. L. S. de, \& Bornstein, M. $\mathrm{H}$. (2003). Socioeconomic status in Brazilian psychological research: I. validity, measurement, and application. Estudos de Psicologia (Natal), 8(3), 375-383. https://doi.org/10.1590/S1413-294X2003000300004

Roberts, J. E., McCary, L. M., Shinkareva, S. V., \& Bailey, D. B. (2016). Infant Development in Fragile X Syndrome: Cross-Syndrome Comparisons. Journal of Autism and Developmental Disorders, 46(6), 20882099. https://doi.org/10.1007/s10803-016-2737-1 
Rowe, M. L., Denmark, N., Jones, H. B., \& Stapleton, L. M. (2016). The Role of Parent Education and Parenting Knowledge in Children's Language and Literacy Skills among White, Black, and Latino Families. Infant and Child Development, 25(2), 198-220. https://doi. org/10.1002/icd.1924

Saccani, R., Valentini, N. C., Pereira, K. R., Muller, A. B., \& Gabbard, C. (2013). Associations of biological factors and affordances in the home with infant motor development. Pediatrics International, 55(2), 197-203. https://doi.org/10.1111/ped.12042

Safadi, R. R., Ahmad, M., Nassar, O. S., Alashhab, S. A. Abdelkader, R., \& Amre, H. M. (2016). Jordanian mothers' knowledge of infants' childrearing and developmental milestones. International Nursing Review, 63(1), 50-59. https://doi.org/10.1111/inr.12185

Scarzello, D., Arace, A., \& Prino, L. E. (2016). Parental practices of Italian mothers and fathers during early infancy: The role of knowledge about parenting and child development. Infant Behavior and Development, 44, 133-143. https://doi.org/10.1016/j. infbeh.2016.06.006

September, S. J., Rich, E., \& Roman, N. (2017). Association Between Knowledge of Child Development and Parenting: A Systematic Review. The Open Family Studies Journal, 9(1), 1-14. https://doi. org/10.2174/1874922401709010001

Shaffer, D. R., \& Kipp, K. (2012). Psicologia do desenvolvimento: infância e adolescência (2nd ed.). São Paulo: Cengage Learning. (DOI INEXISTENTE)

Silva, A. K., Vieira, M. L., Moura, M. L. S., \& Ribas Jr., R. de C. (2005). Conhecimento de mães primíparas sobre desenvolvimento infantil. Um estudo em Itajai SC. Rev. bras. Crescimento desenvolv. humano, 15(3). https://doi.org/01-10. 10.7322/jhgd.19766

Stevens, J. H. (1984). Child development knowledge and parenting skills. Family Relations, 33(2), 237-244. https://doi.org/10.2307/583789

Tabachnick, B. G., \& Fidel, L. S. (2013). Using Multivariate Statistics (6thed). Boston, Massachusetts: Allyn and Bacon. (DOI INEXISTENTE)

Vieira, V. A. (2009). Moderação, mediação, moderadora-mediadora e efeitos indiretos em modelagem de equações estruturais: uma aplicação no modelo de desconfirmação de expectativas. Revista de Administração Universidade de São Paulo RAUSP, 44(1), 17-33. (DOI INEXISTENTE)

Wacharasin, C., Barnard, K., \& Spieker, S. J. (2003). Factors Affecting Toddler Cognitive Development in Low-Income Families: Implications for Practitioners. Infants and Young Children, 16(2), 175-181. https://doi. org/10.1097/00001163-200304000-00009

Yue, A., Wang, X., Yang, S., Shi, Y., Luo, R., Zhang, Q., ...Rozelle, S. (2017). The relationship between infant peer interactions and cognitive development: Evidence from rural China. Chinese Journal of Sociology, 3(2), 193-207. https://doi.or$\mathrm{g} / 10.1177 / 2057150 \times 17702091$

\section{Endereço para correspondência}

Patricia Alvarenga (Instituto de Psicologia, Universidade Federal da Bahia. Rua Aristides Novis, 197, Estrada de São Lázaro. Salvador, Bahia, Brasil, CEP.: 40210-730; Telefone: +55 713283 6480; E-mail: palvarenga66@gmail.com).

\section{Autor I}

Nome: Patricia Alvarenga

Titulação Acadêmica: Doutorado em Psicologia

Afiliação Institucional: Universidade Federal da Bahia (UFBA)

Autor II

Nome: Zelma Freitas Soares

Titulação Acadêmica: Mestranda em Psicologia

Afiliação Institucional: Universidade Federal da Bahia (UFBA)

\section{Autor III}

Nome: Paula Kleize Costa Sales

Titulação Acadêmica: Mestranda em Psicologia

Afiliação Institucional: Universidade Federal da Bahia (UFBA)

\section{Autor IV}

Nome: Nilton Correia dos Anjos Filho

Titulação Acadêmica: Mestrando em Psicologia

Afiliação Institucional: Universidade Federal da Bahia (UFBA) 